\title{
On Strongly F - Regular Modules and Strongly Pure Intersection Property
}

\author{
Bahar H. Al-Bahrani* \\ Received 17, February, 2013 \\ Accepted 8, September, 2013
}

\begin{abstract}
:
A submodule $A$ of amodule $M$ is said to be strongly pure, if for each finite subset $\left\{a_{i}\right\}$ in $\mathrm{A}$, (equivalently, for each $\mathrm{a} \in \mathrm{A}$ ) there exists ahomomorphism $\mathrm{f}: \mathrm{M} \rightarrow \mathrm{A}$ such that $\mathrm{f}\left(\mathrm{a}_{\mathrm{i}}\right)=\mathrm{a}_{\mathrm{i}}, \forall \mathrm{i}(\mathrm{f}(\mathrm{a})=\mathrm{a})$.

A module $\mathrm{M}$ is said to be strongly $\mathrm{F}$-regular if each submodule of $\mathrm{M}$ is strongly pure . The main purpose of this paper is to develop the properties of strongly $\mathrm{F}$-regular modules and study modules with the property that the intersection of any two strongly pure submodules is strongly pure .
\end{abstract}

Key words: Strongly pure submodule ,Strongly F-regular module , Idempotent submodule, Fully idempotent module .

\section{Introduction :}

All rings are commutative with identity element and all modules are unitary left modules, unless otherwise stated . Following [1] a submoduleA of a module is called strongly pure if for each finite subset $\left\{a_{i}\right\}$ in $A$, (equivalently, for each $a \in A$ ) there exists ahomomorphism $\mathrm{f}: \mathrm{M} \rightarrow \mathrm{A}$ such that $\mathrm{f}\left(\mathrm{a}_{\mathrm{i}}\right)=\mathrm{a}_{\mathrm{i}}, \forall \mathrm{i}$.

$M$ is $Z$ regular if for each a $\in M, \exists f$ $\in \mathrm{M}^{*}=\operatorname{Hom}(\mathrm{M}, \mathrm{R})$ such that $\mathrm{a}=\mathrm{f}(\mathrm{a})$ a . Equivalently, each f.g. submodule of $\mathrm{M}$ is projective direct summand [1] . $\mathrm{M}$ is $\mathrm{F}$ - regular if eachsubmodule of $\mathrm{M}$ is pure.

It is known that if $\mathrm{N}$ is a finitely generated strongly pure submodule of $\mathrm{M}$, then $\mathrm{N}$ is a summand [1] . Clearly that every strongly pure submodule of a module $\mathrm{M}$ is pure, The converse is true if $\mathrm{M}$ is projective [1] .

Note that a ring $\mathrm{R}$ is $\mathrm{Z}$-regular module iff $\mathrm{R}$ is strongly $\mathrm{F}$ - regular iff $\mathrm{R}$ is $\mathrm{F}-$ regular module iff $R$ is a regular ring (in the sense of Von Neumann) [1] .

Let $\mathrm{R}$ be an associative ring withidentity, and let $\mathrm{M}$ be a (left) unitary module . Following [2] a submoduleA of a module $M$ is called idempotent submodule of $\mathrm{M}$ provided $\mathrm{N}=\operatorname{Hom}(\mathrm{M}, \mathrm{A}) \quad \mathrm{A}=$ $\sum\{f(A) ; f: M \rightarrow A\}$. That is $\mathrm{A}$ is an idempotent submoduleof $M$ if for each $\mathrm{x} \in \mathrm{N}$, there exist a positive integer $\mathrm{k}$, homomorphismsf $f_{\mathrm{i}}: \mathrm{M} \rightarrow \mathrm{A} \quad(1 \leq \mathrm{i} \leq \mathrm{k})$ such that $\mathrm{x}=\mathrm{f}_{1}\left(\mathrm{x}_{1}\right)+\ldots .+\mathrm{f}_{\mathrm{k}}\left(\mathrm{x}_{\mathrm{k}}\right)$.

Clearly every strongly pure submodule is an idempotent submodule, The converse is not true . A module $\mathrm{M}$ is said to be fully idempotent if every submodule of $\mathrm{M}$ is idempotent .

In [3] ,Naoum, A. G. Al - HashimiB. A. and $\mathrm{Al}$ - Bahrani , B.H. studied modules with the property that the intersection of any two pure submodules is pure (PIP). This led us to introduce the concept of a module with the property that the intersection of any two strongly pure submodules is strongly pure (STPIP) .

In section 1 we study strongly $\mathrm{F}-$ regular. We prove that a module $\mathrm{M}$ is strongly F-regular iff every essential submodule of $\mathrm{M}$ is strongly pure, see

*Department of Mathematics, College of Science, University of Baghdad 
prop 1.6. Also we prove that amodule $M$ is fully idempotent iff for every submodule $A$ of $\mathrm{M}$ and for every homomorphism $0 \neq \mathrm{f} \in \operatorname{Hom}(\mathrm{A}, \mathrm{L})$ where $\mathrm{L}$ is any module, there exists a homomorphism $\mathrm{g} \in \operatorname{Hom}(\mathrm{M}, \mathrm{M})$ such that $\mathrm{f} g(\mathrm{~A}) \neq 0$, see prop1.11.

Insection 2 of the paper we study modules with the property that the intersection of any two strongly pure submodules is strongly pure. We prove that if $\mathrm{M}$ is a module with the STPIP. Then for every decomposition $\mathrm{M}=\mathrm{A} \oplus \mathrm{B}$ and for every $\mathrm{R}-$ homomorphism $\mathrm{f}: \mathrm{A} \rightarrow \mathrm{B}, \quad$ ker $\mathrm{f}$ is strongly pure in $\mathrm{M}$.

\section{Strongly $F$ - regular modules}

First we recall some basic properties of strongly pure submodules .

Lemma 1.1 [1]. Let $\mathrm{M}$ be an $\mathrm{R}$-module and let $A, B$ be submodules of $M$ such that $\mathrm{A} \subseteq \mathrm{B}$.

1) If $A$ is a strongly pure submodule of $M$, then $A$ is a strongly pure sub module of $\mathrm{B}$.

2) If $A$ is a strongly pure submodule of $\mathrm{B}$ and $\mathrm{B}$ is a strongly pure sub module of $\mathrm{M}$, then $\mathrm{A}$ is a strongly pure submodule of $\mathrm{M}$.

3) If $\mathrm{A}$ is a fully invariant submodule of $\mathrm{M}$ and $\mathrm{B}$ is a strongly puresubmodule of $\mathrm{M}$, then $\frac{B}{A}$ is a strongly pure submodule of $\frac{M}{A}$.

Proof .clear

Lemma1.2 [1] Every f.g strongly pure submodule is a direct summand .

Proof . Let $\mathrm{A}=\mathrm{Ra}_{1}+\ldots .+\mathrm{Ra}_{\mathrm{k}} \mathrm{be} \mathrm{a}$ strongly pure submodule of a module $M$. Then there exist a homomorphism $\mathrm{f}: \mathrm{M} \rightarrow$ A such that $\mathrm{f}\left(\mathrm{a}_{\mathrm{i}}\right)=\mathrm{a}_{\mathrm{i}}, \forall 1 \leq \mathrm{i} \leq$ k. Thus $\mathrm{f}(\mathrm{a})=\mathrm{a}, \forall \mathrm{a} \in \mathrm{A}$. Clearly $\mathrm{f}$ is a split epimorphism .Thus $\mathrm{A}$ is a direct summand of $\mathrm{M}$, by [4] .
Lemma 1.3Let $M=A \oplus B$ be $a$ torsion free module. Then $\mathrm{R}(\mathrm{a}+\mathrm{b})$ is strongly pure in $\mathrm{Ra} \oplus \mathrm{Rb}$, for every a $\in A$ and $b \in B$. Hence $R(a+b)$ is $a$ direct summand of $\mathrm{Ra} \oplus \mathrm{Rb}$.

Proof . Let $f: R a \oplus R b \rightarrow R(a+b)$ be $a$ map defined by $f\left(r_{1} a+r_{2} b\right)=r_{1}(a+b)$. Clearly $\mathrm{f}$ is a homomorphism and $\mathrm{f}(\mathrm{a}+$ $\mathrm{b})=\mathrm{a}+\mathrm{b}$. Thus $\mathrm{R}(\mathrm{a}+\mathrm{b})$ is strongly pure in $\mathrm{Ra} \oplus \mathrm{Rb}$.

By Lemma $1.2, \mathrm{R}(\mathrm{a}+\mathrm{b})$ is a direct summand of $\mathrm{Ra} \oplus \mathrm{Rb}$.

Lemma 1.4 . Let $A$ and $B$ be submodules of a module $M$ such that $\mathrm{A} \subseteq \mathrm{B}$.If $\mathrm{A}$ is strongly pure in $\mathrm{M}, \frac{B}{A}$ is strongly pure in $\frac{M}{A}$ and $M$ is $\mathrm{B}$ projective, then $\mathrm{B}$ is strongly pure in M .

Proof . Let $x \in B$, then there exist a homomorphism $\mathrm{f}: \frac{M}{A} \rightarrow \frac{B}{A}$ such that $\mathrm{f}(\mathrm{x}$ $+\mathrm{A})=\mathrm{x}+\mathrm{A}$. Now consider the following diagram

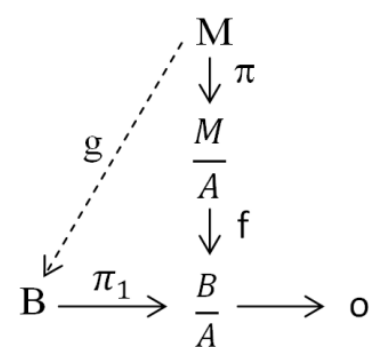

Where $\pi$ and $\pi_{1}$ are the natural epimorphisms. Since $\mathrm{M}$ is $\mathrm{B}$ projective, then there exist a homomorphism $\mathrm{g}: \mathrm{M} \rightarrow \mathrm{B}$ such that $\pi_{1} g=f \pi$. So $g(x)+A=f(x+A)=x+$ $A$. Thus $x-g(x) \in A$. But $A$ is strongly pure in $\mathrm{M}$, therefore there is a homomorphism $\mathrm{h}: \mathrm{M} \rightarrow \mathrm{A}$ such that $\mathrm{h}(\mathrm{x}$ $-\mathrm{g}(\mathrm{x}))=\mathrm{x}-\mathrm{g}(\mathrm{x})$. Hence $\mathrm{x}=\mathrm{h}(\mathrm{x})-\mathrm{hg}(\mathrm{x})$ $+g(x)=(h-h g+g)(x)$. Now consider the homomorphism $\mathrm{k}=(\mathrm{ih}-\mathrm{ihg}+\mathrm{g})$ : $\mathrm{M} \rightarrow \mathrm{B}$, where iis inclusion map. Thus $\mathrm{B}$ is strongly pure in $\mathrm{M}$. 
Proposition 1.5 . The following statements are equivalent for a module M .

1) $M$ is strongly $F-$ regular .

2) $\mathrm{Rm}$ is strongly pure in $\mathrm{M} . \forall \mathrm{m} \in \mathrm{M}$.

3) $\mathrm{Rm}$ is a direct summand of $\mathrm{M}, \forall \mathrm{m}$ $\in \mathrm{M}$.

Proof .Clear .

Proposition 1.6. A module $M$ is strongly $\mathrm{F}$-regular iff every essential submodule of $\mathrm{M}$ is strongly pure in $\mathrm{M}$.

Proof $\rightarrow$ ) clear

$\leftarrow)$ Let $\mathrm{A}$ be any submodule of $\mathrm{M}$ and $\mathrm{B}$ be a relative complment of $\mathrm{A}$ in $\mathrm{M}$. Then by [5], $\mathrm{A} \oplus \mathrm{B}$ is essential in $\mathrm{M}$. So $\mathrm{A} \oplus \mathrm{B}$ is strongly pure in $\mathrm{M}$. But $\mathrm{A}$ is strongly pure in $A \oplus B$, therefore $A$ is strongly pure in $\mathrm{M}$, by lemma (1.1 2) .

Lemma 1.7 . Let $M$ be a f.g strongly $F$ - regular module and End(M) be the endomorphism ring of $M$. Then for every $f \in \operatorname{End}(M), f(M)$ is a direct summand of $\mathrm{M}$.

Proof . Since $M$ is f.g and $f(M) \simeq \frac{M}{\operatorname{ker} f}$ , then $f(M)$ is f.g submodule of $M$. Thus $\mathrm{f}(\mathrm{M})$ is a direct summand of $\mathrm{M}$, by lemma 1.2 .

Let $\mathrm{M}$ be a module . $\mathrm{M}$ is called a multiplication module if each submodule $\mathrm{N}$ of $\mathrm{M}$ has the form IM for some ideal I of R[1] .

Proposition 1.8. Let $M$ be a f.g faithful multiplication module. If $\mathrm{M}$ is strongly $\mathrm{F}$-regular, then $\mathrm{R}$ is regular .

Proof . Let $a \in R$ and $f: M \rightarrow a M$ be the epimorphism defined by $\mathrm{f}(\mathrm{m})=\mathrm{am}$. Since $M$ is f.g and $\frac{M}{\operatorname{ker} f} \simeq \mathrm{aM}$, then aM is f.g and hence a direct summand of $\mathrm{M}$, by Lemma 1.2 . Thus $\mathrm{M}=\mathrm{aM} \oplus \mathrm{B}$ , for some submodule B of M. Since
$\mathrm{M}$ is multiplication, then $\mathrm{B}=\mathrm{IM}$, for some ideal $I$ of $R$. Now $M=R M=$ (a) $\mathrm{M} \oplus \mathrm{IM}=((\mathrm{a}) \oplus \mathrm{I}) \mathrm{M}$.But $\mathrm{M}$ is a cancellation module, by [6] . Thus $\mathrm{R}=$ (a) $\oplus \mathrm{I}$ and hence $\mathrm{R}$ is regular .

Let $\mathrm{R}$ be an associative ring with identity and let $\mathrm{M}$ be a module . In [2] A submodule $\mathrm{A}$ of $\mathrm{M}$ is called idempotent if $\mathrm{A}=\operatorname{Hom}(\mathrm{M}, \mathrm{A}) \mathrm{A}=$ $\sum\{f(\mathrm{~A}): \mathrm{f}: \mathrm{M} \rightarrow \mathrm{A}\}$. That is $\mathrm{A}$ is idempotent in $M$ if, for each $x \in A$, there exist a positive integer $\mathrm{k}$, homomorphismsf $f_{\mathrm{i}}: \mathrm{M} \rightarrow \mathrm{A}(1 \leq \mathrm{i} \leq \mathrm{k})$ and elements $x_{i} \in A(1 \leq i \leq k)$ such that $x=$ $\mathrm{f}_{1}\left(\mathrm{x}_{1}\right)+\ldots .+\mathrm{f}_{\mathrm{n}}\left(\mathrm{x}_{\mathrm{n}}\right)$. In [2], $\mathrm{M}$ is called fully idempotent if every submodule of $\mathrm{M}$ is idempotent in $\mathrm{M}$.

Now ,If $M$ is a module over a commutative ringwith1. Then Clearly that every strongly pure submodule of $\mathrm{M}$ is an idempotent .The converse is not true in general. For example let $\mathrm{Z}$ be the ring of integers . By ([2], Coro 2.9) The sub module $A=(2,0) Z \oplus(1,1)$ $\mathrm{Z}$ is an idempotent sub module of the free module $Z \oplus Z$. Claim that $A$ is not strongly pure in $\mathrm{Z} \oplus \mathrm{Z}$.If not, then $\mathrm{A}$ is a direct summand of $Z \oplus Z$, by lemma 1.2 .

Thus $(2,0) Z=2 Z \oplus 0$ is a direct summand of $\mathrm{Z} \oplus \mathrm{Z}$ which is a contradiction (since $2 \mathrm{Z}$ is not a direct summand of Z) .

Now we give some results on idempotent submodules.

Proposition 1.9. Let $\mathrm{R}$ be an associative ring with 1 . Let $\mathrm{A}$ be a submodule of a module $M$. If for each $\mathrm{x} \in \mathrm{A}$, there exist a positive integer $\mathrm{k}$, homomorphisms $\mathrm{f}_{\mathrm{i}} \in \operatorname{Hom}(\mathrm{M}, \mathrm{R}) \quad(1 \leq \mathrm{i} \leq$ k) and elements $x_{i} \in A(1 \leq i \leq k)$ such that $\mathrm{x}=\mathrm{f}_{1}\left(\mathrm{x}_{1}\right) \mathrm{x}_{1}+\ldots .+\mathrm{f}_{\mathrm{k}}\left(\mathrm{x}_{\mathrm{k}}\right) \mathrm{x}_{\mathrm{k}}$, then $\mathrm{A}$ is an idempotent submodule of $\mathrm{M}$.

Proof . For each $(1 \leq \mathrm{i} \leq \mathrm{k})$, Let $\mathrm{g}_{\mathrm{i}}: \mathrm{R}$ $\rightarrow \mathrm{Rx}_{\mathrm{i}}$ be the homomorphism defined 
by $\mathrm{g}_{\mathrm{i}}(\mathrm{r})=\mathrm{rx}_{\mathrm{i}}$ and $\mathrm{j}_{\mathrm{i}}: \mathrm{Rx}_{\mathrm{i}} \rightarrow \mathrm{A}$ be the inclusion map .

So $, \mathrm{h}_{\mathrm{i}}=\mathrm{j}_{\mathrm{i}} \mathrm{g}_{\mathrm{f}} \mathrm{f}_{\mathrm{i}}: \mathrm{M} \rightarrow \mathrm{A}$ is a homomorphism and $\mathrm{x}=\mathrm{h}_{1}\left(\mathrm{x}_{1}\right)+\ldots .+$ $\mathrm{h}_{\mathrm{k}}\left(\mathrm{x}_{\mathrm{k}}\right)$. Thus $\mathrm{A}$ is an idempotent submodule of $\mathrm{M}$.

Proposition 1.10. Let $\mathrm{R}$ be an associative ring with 1 and $\mathrm{A}$ be an idempotent submodule of a module $M$. Then $\operatorname{Hom}(\mathrm{M}, \mathrm{A})$ is an ideal of End(M) the endomorphism ring of $\mathrm{M}$ iff $\mathrm{A}$ is a fully invariant submodule of $\mathrm{M}$.

Proof. Let $g \in \operatorname{End}(M)$. Since $A=$ $\operatorname{Hom}(\mathrm{M}, \mathrm{A}) \mathrm{A}=\Sigma\{f(A) ; \mathrm{f}: \mathrm{M} \rightarrow \mathrm{A}\}$, then $\mathrm{g}(\mathrm{A})=\mathrm{g}\left(\sum f(A)\right)=\sum\{g f(A) ; \mathrm{f}$ : $\mathrm{M} \rightarrow \mathrm{A}$ \}. But $\operatorname{Hom}(\mathrm{M}, \mathrm{A})$ is an ideal in $\operatorname{End}(\mathrm{M})$, therefore $\mathrm{gf} \in \operatorname{Hom}(\mathrm{M}, \mathrm{A})$, $\forall \mathrm{f} \in \operatorname{Hom}(\mathrm{M}, \mathrm{A})$ and hence $\mathrm{g}(\mathrm{A}) \subseteq \mathrm{A}$. Thus $\mathrm{A}$ is a fully invariant submodule of $\mathrm{M}$.

The converse ,Let $g \in \operatorname{End}(M)$ and $\mathrm{f} \in \operatorname{Hom}(\mathrm{M}, \mathrm{A})$. Since $A$ is fully invariant in $\mathrm{M}$, then $(\mathrm{gf})(\mathrm{A}) \subseteq \mathrm{A}$ and $(f g)(A) \subseteq A$. So $\operatorname{Hom}(M, A)$ is an ideal of $\operatorname{Hom}(\mathrm{M}, \mathrm{A})$.

Recall that an $\mathrm{R}$ - module $\mathrm{M}$ is called fully idempotent if every submodule of $\mathrm{M}$ is idempotent , [2] .

Now, we give a characterization for fully idempotent modules .

Proposition 1.11. Let $\mathrm{M}$ be a module over associative ring with 1.A module $M$ is fully idempotent iff for every submodule $A$ of $M$ and every 0 $\neq \mathrm{g} \in \operatorname{Hom}(\mathrm{A}, \mathrm{L})$, where $\mathrm{L}$ is any module, there exists $\mathrm{h} \in \operatorname{Hom}(\mathrm{M}, \mathrm{A})$ such that $\operatorname{gh}(\mathrm{A}) \neq 0$

Proof . Let $0 \neq \mathrm{g} \in \operatorname{Hom}(\mathrm{A}, \mathrm{L})$ and $\mathrm{x} \in$ A such that $g(x) \neq 0$. Then there exist a positive integer $\mathrm{k}$, homomorphismsf $\mathrm{f}_{\mathrm{i}}$ : $\mathrm{M} \rightarrow \mathrm{A}(1 \leq \mathrm{i} \leq \mathrm{k})$ such that $\mathrm{x}=\mathrm{f}_{1}\left(\mathrm{x}_{1}\right)+$ $\ldots+\mathrm{f}_{\mathrm{k}}\left(\mathrm{x}_{\mathrm{k}}\right)$.

If $\mathrm{g}_{\mathrm{i}}(\mathrm{A})=0, \forall 1 \leq \mathrm{i} \leq \mathrm{k}$, then $\mathrm{g}(\mathrm{x})=0$ which is a contradiction .
So $g \mathrm{f}_{\mathrm{i}}(\mathrm{A}) \neq 0$, for some $1 \leq \mathrm{i} \leq \mathrm{k}$ and $\mathrm{f}_{\mathrm{i}}$ is the required homomorphism .

The converse . Let $\mathrm{a} \in \mathrm{M}$ and put $\mathrm{A}=\sum\{f(R a) ; \mathrm{f} \quad: \mathrm{M} \rightarrow \mathrm{Ra}\}=$ $\operatorname{Hom}(\mathrm{M}, \mathrm{Ra}) \mathrm{Ra}$. Clearly that $\mathrm{A} \subseteq \mathrm{Ra}$. Claim that $A=R a$.If $A \neq R a$, Let $\pi$ : Ra $\rightarrow \frac{R a}{A}$ be the natural epimorphism.Clearly that $\pi \neq 0$.So there exist $\mathrm{h} \in \operatorname{Hom}(\mathrm{M}, \mathrm{Ra})$ such that $(\pi \mathrm{h})(\mathrm{Ra}) \neq 0$. So $\mathrm{h}(\mathrm{Ra}) \not \subset \mathrm{A}$ which is a contradiction . Thus $A=R a=\operatorname{Hom}(M$, Ra)Ra.By ([2] , Lemma 2.15) $\mathrm{M}$ is fully idempotent .

Recall that module $\mathrm{M}$ is said to have the summand sum property (SSP) if the sum of any two direct summand is again a direct summand [7] .

Proposition 1.12Let $M$ be a module over associative ring with 1 . If $M$ is fully idempotent and $\oplus_{I} M$ has SSP, for every index set $I$, then $M$ is semisimple.

Proof.let $\mathrm{A}$ be a submodule of $\mathrm{M}$. since $\mathrm{A}$ is idempotent in $\mathrm{M}$, then there exists a family of R-homomorphisms $\left\{f_{\alpha} \mid f_{\alpha} \in \operatorname{Hom}(M, A), \forall_{\alpha} \in \Lambda\right\}$ such that $A=\sum\left\{f_{\alpha}(A) \mid \alpha \in \Lambda\right\}$. define $f: \oplus_{\alpha \in \Lambda} M \rightarrow A$ by $f\left(\left(m_{\alpha}\right)_{\alpha \in A}\right)=\sum_{\alpha \in A} f_{\alpha}\left(m_{\alpha}\right)$. Clearly that $\mathrm{f}$ is an epimorphism. Let $\mathrm{i}: \mathrm{A} \rightarrow \mathrm{M}$ he the inclusion map. Since $\left(\oplus_{\alpha \in M} M\right) \oplus M$ has SSP, then by [7] $\operatorname{lm}$ if $=A$ is a direct summand of $M$. Thus $\mathbf{M}$ is semisimple.

Proposition 1.13.let I be an ideal of an associative ring $\mathrm{R}$ with 1 . If $\mathrm{I}$ is a pure ideal of $\mathrm{R}$, then $\mathrm{I}$ is idempotent. The converse is true if I is fully idempotent

Proof . Let I be a pure ideal of $\mathrm{R}$.then for every ideal $\mathrm{J}$ of $\mathrm{R}, \mathrm{J} . \mathrm{I}=\mathrm{J} \cap \mathrm{I}$ and hence $I^{2}=I$. Thus $I$ is an idempotent ideal of $\mathrm{R}$, by [2]. 
The converse, Let $t \in I$, there exist a positive integer $\mathrm{k}$, homomorphisms $f_{i}: I \rightarrow R t(1 \leq i \leq k)$ and elements $r_{i} \in R(1 \leq i \leq k)$ such that $\mathrm{t}=$ $r_{1} f_{1}(t)+\cdots \ldots \ldots+r_{k} f_{k}(t)$, by ([2] lemma 2.15). Since $\mathrm{t} \in \mathrm{I}=\mathrm{I}^{2}$, then $\mathrm{t}=$ $\sum_{j=1}^{n} \alpha_{j} b_{j} \quad$, where $\alpha_{j}, b_{j} \in I(\forall 1 \leq j \leq n)$.now $\mathrm{t} \quad=$ $\sum_{i=1}^{k} r_{i} f_{i}\left(\sum_{j=1}^{n} a_{j} b_{j}\right)=$

$\sum_{i=1}^{k} r_{i} \sum_{j=1}^{n} a_{j} f_{i}\left(b_{j}\right)$ Let $f_{i}\left(b_{j}\right)=S_{i j} t$, where $S_{i j} \in R(\forall 1 \leq i \leq k, 1 \leq j \leq n)$ So $\mathrm{t}=\sum_{i=1}^{k} r_{i} \sum_{j=1}^{n} a_{j} s_{i j} t=$ $\left(\sum_{i=1}^{k} \sum_{j=1}^{n} r_{i} a_{j} S_{i j}\right) t$. Let $\mathrm{S}=$ $\left(\sum_{i=1}^{k} \sum_{j=1}^{n} r_{i} a_{j} S_{i j}\right) \in I$. Thus $\mathrm{t}=\mathrm{st}$ and $\mathrm{I}$ is a pure ideal.

Proposition 1.14[2]. Let $M$ be a module over a commutative ring . Then $\mathrm{M}$ is fully idempotent iff every cyclic Submodule of $\mathrm{M}$ is a direct summand .

Proposition 1.15. Let $\mathrm{R}$ he a commutative ring. Then an $\mathrm{R}$ - module $\mathrm{M}$ is fully idempotent if $\mathrm{f} \mathrm{M}$ is strongly $\mathrm{F}-$ regular.

Proof .clear by Prop. 1.5

Theorem 1.15 [2]. The following are equivalent for a commutative ring :

1. Every R- module is fully idempotent .

2. Every injective $\mathrm{R}$ - module is fully idempotent.

3. Every cyclic R - module is injective .

4. $\quad \mathrm{R}$ is semisimple.

2. Module with the Strongly Pure Intersection Property.

In this section we introduce the concept of the strongly pure intersection property for modules (STPIP) , and give some basic Properties. We start by a definition .

\section{Definition 2.1.}

A module has the strongly pure intersection property (briefly STPIP) if the intersection of any two strongly pure submodules is again strongly pure.

Recall that module $\mathrm{M}$ is called strongly pure Simple if $\mathrm{O}$ and $\mathrm{M}$ are the only Strongly pure Submodules of $\mathrm{M}$. Clearly that every strongly pure simple module has the STPIP.For example $\mathrm{Z}$ as $\mathrm{Z}$ - module.

Also every strongly $\mathrm{F}$ - regular module satisfies the STPIP trivially.

The following example show that the intersection of two strongly pure submodules need not be strongly pure.

Example 2.2.Consider the module $M$ $=\mathrm{Z}_{4} \oplus \mathrm{Z}_{2}$ as $\mathrm{Z}-$ module. Let $\mathrm{A}=\mathrm{Z}_{4} \oplus 0$ and $B=Z(1,1)$. It is clear that $A$ and $B$ are direct Summand of $M$. But $A \cap B$ $=\{(0,0),(2,0)\}$ is not a direct summand of $\mathrm{M}$. Hence $\mathrm{A} \cap \mathrm{B}$ is not strongly pure in $\mathrm{M}$, by lemma 1.2.

\section{Proposition 2.3.}

If a module $M$ has the STPIP, then every strongly pure submodule A of $\mathrm{M}$ has the STPIP,

Proof.clear , by Lemma 1.1

Proposition 2.4. Let $M$ be a quasi projective module and has the STPIP. If $\mathrm{A}$ is a strongly pure submodule of $\mathrm{M}$ and fully invariant, then $\frac{M}{A}$ has the STPIP.

Proof : Let $\frac{C}{A}$ and $\frac{D}{A}$ be strongly pure submodules of $\frac{M}{A}$. Since $\mathrm{M}$ is $\mathrm{M}-$ projective, then by [8] $\mathrm{M}$ is $\mathrm{C}$ projective and $\mathrm{M}$ is $\mathrm{D}$ - projective. So by Lemma 1.4. , C and D are strongly Pure in M.Hence $\mathrm{C} \cap \mathrm{D}$ is strongly pure in M. To show that $\frac{C}{A} \cap \frac{D}{A}=\frac{C \cap D}{A}$ is strongly Pure in $\frac{M}{A}$, Let $\mathrm{x}+\mathrm{A} \in \frac{C \cap D}{A}, \mathrm{x}$ $\in \mathrm{C} \cap \mathrm{D}$. So there exists a homomorphism $\mathrm{f}: \mathrm{M} \rightarrow \mathrm{C} \cap \mathrm{D}$ Such 
that $\mathrm{f}(\mathrm{x})=\mathrm{x}$. Let $f^{\prime}: \frac{M}{A} \rightarrow \frac{C \cap D}{A}$ be a map defined by $f^{\prime}(m+A)=f(m)+A$. Since A is fully Invariant, then $f^{x}$ is well define. Clearly that $f^{\prime}(x+A)=x+A$. Thus $\frac{M}{A}$ has STPIP.

\section{Proposition 2.5.}

Let $M$ be a module.If the endomorphism ring $\operatorname{End}(\mathrm{M})$ is commutative, then $\mathrm{M}$ has the STPIP.

Proof: Let A and B be strongly pure submodules of $M$ and $x \in A \cap B$.So there exist homomorphismsf : $\mathrm{M} \rightarrow \mathrm{A}$ and $\mathrm{g}: \mathrm{M} \rightarrow \mathrm{B}$ such that $\mathrm{f}(\mathrm{x})=\mathrm{x}$ and $\mathrm{g}(\mathrm{x})=\mathrm{x}$. Now, we can consider $\mathrm{gf}$, $\mathrm{fg} \in \operatorname{End}(\mathrm{M})$. Since $\mathrm{E}(\mathrm{M})$ is commutative, then gf $=$ fg. But $(\mathrm{gf})$ (M) $\subseteq \mathrm{A} \cap$ B. So there exist the homomorphism igf : $\mathrm{M} \rightarrow \mathrm{A} \cap \mathrm{B}$ such that (igf) ( $\mathrm{x})=\mathrm{x}$, where $\mathrm{i}$ is the inclusion map. Thus M has the STPIP .

Corollary : 2.6.every multiplication module has the STPIP. In particular every commutative ring with identity has the STPIP as R-module.

\section{Proof .Clear by [1]}

Recall that an $\mathrm{R}$ - module $\mathrm{M}$ is a Quasi - Dedekind module if every non zero endomorphism of $\mathrm{M}$ is $\mathrm{a}$ monomorphism [9] .

Proposition 2.8 . Every Quasi Dedekind module is strongly pure simple. Hence has the STPIP .

Proof . Let $0 \neq \mathrm{A}$ be a strongly pure submodule of $M$ and $0 \neq a \in A$.

So there is a homomorphism $\mathrm{f}: \mathrm{M} \rightarrow$ A such that $\mathrm{f}(\mathrm{a})=\mathrm{a}$.

Now consider the homomorphism $1-\mathrm{f}$ $: \mathrm{M} \rightarrow \mathrm{M}$. $(1-\mathrm{f})(\mathrm{a})=0$.

So $0 \neq a \in \operatorname{ker}(1-f)$ which is a contradiction. Thus $\mathrm{f}=1$ and $\mathrm{A}=\mathrm{M}$.
The following theorem is the main tool for our subsequent results

Theorem 2.9. If a module $M$ has the STPIP , then for every decomposition $\mathrm{M}=\mathrm{A} \oplus \mathrm{B}$ and every homomorphism $\mathrm{f}: \mathrm{A} \rightarrow \mathrm{B}$, ker $\mathrm{f}$ is a strongly pure submodule of $\mathrm{M}$.

Proof . Let $T=\{a+f(a): a \in A\}$. To show that $\mathrm{M}=\mathrm{T} \oplus \mathrm{B}$,

Let $x \in M$, then $x=a+b, a \in A, b \in B$ . So $x=a+f(a)-f(a)+b, a+f(a) \in$ $\mathrm{T}, \mathrm{f}(\mathrm{a})+\mathrm{b} \in \mathrm{B}$. Now let $\mathrm{x} \in \mathrm{T} \cap \mathrm{B}$. Hence $x=a+f(a), a \in A$. So $a=x-$ $\mathrm{f}(\mathrm{a}) \in \mathrm{A} \cap \mathrm{B}=0$. Thus $\mathrm{x}=0$. Since $\mathrm{M}$ has the STPIP, then $\mathrm{T} \cap \mathrm{A}$ is strongly pure in M.It is easy to show that ker $\mathrm{f}=$ $\mathrm{T} \cap \mathrm{A}$. Thus ker $\mathrm{f}$ is a strongly pure sub module of $\mathrm{M}$.

Proposition 2.10 . Let $M$ be a strongly pure simple module and Let $\mathrm{N}$ be any module. If $\mathrm{M} \oplus \mathrm{N}$ has the STPIP, then either $\operatorname{Hom}(\mathrm{M}, \mathrm{N})=0$ or every non zero homomorphism from $\mathrm{M}$ to $\mathrm{N}$ is a monomorphism .

Proof . Assume $\operatorname{Hom}(\mathrm{M}, \mathrm{N}) \neq 0$ and Let $\mathrm{f}: \mathrm{M} \rightarrow \mathrm{N}$ be a non zero homomorphism. Since $M \oplus N$ has the STPIP, then ker $\mathrm{f}$ is strongly pure in $\mathrm{M}$. But $\mathrm{M}$ is strongly pure simple ,Soker $\mathrm{f}=0$ and $\mathrm{f}$ is a monomorphism . The following corollary follows immediately from prop. 2.10.

Corollary 2.11 . Let $M$ be a strongly pure simple module.If $M \oplus M$ has the STPIP , then M is Quasi - Dedekind .

Recall that an $\mathrm{R}$ module $\mathrm{M}$ is called a flat R-module if for any monomorphismf: $\mathrm{A} \rightarrow \mathrm{B}$, where $\mathrm{A}$ and $\mathrm{B}$ are any two R-module, $\mathrm{f} \otimes 1: \mathrm{A} \otimes \mathrm{M}$ $\rightarrow \mathrm{B} \otimes \mathrm{M}$ is a monomorphism, see[10]. 
Proposition 2.12 . Let $\mathrm{M}$ be an $\mathrm{R}-$ module . If $\mathrm{R} \oplus \mathrm{M}$ has the STPIP, then every cyclic submodule of $\mathrm{M}$ is flat .

Proof . Let $m \in M$. Consider the following short exact sequence

$$
0 \rightarrow \operatorname{ker} \mathrm{f} \stackrel{i_{1}}{\rightarrow} \mathrm{R} \stackrel{f}{\rightarrow} \mathrm{R}_{\mathrm{m}} \rightarrow 0
$$

Where $i_{1}$ is the inclusion map and $f$ is defined as follows $\mathrm{f}(\mathrm{r})=\mathrm{rm}, \forall \mathrm{r} \in \mathrm{R}$. Since $\mathrm{R} \oplus \mathrm{M}$ has the STPIP, then by Th. 2.9 ker $\mathrm{f}$ is strongly pure in $\mathrm{R}$. HenceRm is flat by [10] .

The direct sum of two modules with the STPIP may not have the STPIP, See example 2.2.

Now, we give a condition under which the direct sum of modules with the STPIPhas the STPIP.

Proposition 2.13. Let $\mathrm{M}$ and $\mathrm{N}$ be modules with the STPIP such that ann $\mathrm{M}+$ ann $\mathrm{N}=\mathrm{R}$, then $\mathrm{M} \oplus \mathrm{N}$ has the STPIP.

Proof . Let C and D be a strongly pure submodules of $M \oplus N$.Since ann $M+$ ann $\mathrm{N}=\mathrm{R}$, then by the same way of the proof of [11, prop. $(4.2),(4,1)] \mathrm{C}=$ $\mathrm{A} \oplus \mathrm{B}$ and $\mathrm{D}=\mathrm{A}_{1} \oplus \mathrm{B}_{1}$, where $\mathrm{A}$ and $\mathrm{A}_{1}$ are submodules of $\mathrm{M}, \mathrm{B}$ and $\mathrm{B}_{1}$ are submodules of $N$. Since $M$ and $N$ has the STPIP, then $A \cap A_{1}$ is strongly pure in $\mathrm{M}$ and $\mathrm{B} \cap \mathrm{B}_{1}$ is strongly pure in $\mathrm{N}$. One can easily show that $\mathrm{C} \cap \mathrm{D}$ $=\left(\mathrm{A} \cap \mathrm{A}_{1}\right) \oplus\left(\mathrm{B} \cap \mathrm{B}_{1}\right)$ is strongly pure in $\mathrm{M} \oplus \mathrm{N}$. Thus $\mathrm{M} \oplus \mathrm{N}$ has the STPIP.

Theorem 2.14 . Let $\mathrm{R}$ be a ring .If all $\mathrm{R}$ - modules have the STPIP Then all $\mathrm{R}$ - modules are strongly $\mathrm{F}$ - regular.

Proof .Let A be a submodule of an R module $\mathrm{M}$ and Let $\pi: \mathrm{M} \rightarrow \frac{M}{A}$ be the natural epimorphism . By our assumption $\mathrm{M} \oplus \frac{M}{A}$ has the STPIP. Therefore by Th. 2.9, $\operatorname{ker} \pi=\mathrm{A}$ is strongly pure in $M$. Thus $M$ is strongly $\mathrm{F}$-regular .

The converse is clear

Theorem 2.15.Let $\mathrm{R}$ be a ring . Then all injective $\mathrm{R}$ - modules have the STPIP iff all injective $\mathrm{R}$ - modules are strongly $\mathrm{F}$ - regular

Proof .Let $M$ be an injective $\mathrm{R}$ module and $\mathrm{A}$ be a submodule of $\mathrm{M}$ .Let $\pi: \mathrm{M} \rightarrow \frac{M}{A}$ be the natural epimorphism.If $\frac{M}{A}$ is injective, then $\mathrm{M} \oplus \frac{M}{A}$ is injective and hence $\mathrm{M} \oplus \frac{M}{A}$ has the STPIP.Thus, Ker $\pi=\mathrm{A}$ is a strongly pure sub module of $\mathrm{M}$. Thus $\mathrm{M}$ is strongly $\mathrm{F}$ - regular .

Assume $\frac{M}{A}$ is not injectivelet $\left(\frac{M}{A}\right)$ be the injective hull of $\frac{M}{A}$ andi: $\frac{M}{A} \rightarrow\left(\frac{M}{A}\right)$ be the inclusion map. Now consider $\mathrm{i} \pi: \mathrm{M} \rightarrow$ $\left(\frac{M}{A}\right)$. Since $M \oplus\left(\frac{M}{A}\right)$ has the STPIP, then $\operatorname{keri} \pi=\operatorname{ker} \pi=\mathrm{A}$ is strongly pure in $\mathrm{M}$, by Th. 2.9. Thus $\mathrm{M}$ is strongly $\mathrm{F}$ - regular .

The converse is clear .

Theorem 2.16. The following statements are equivalent for a ring $R$

1) $R$ is semisimple .

2) All R - modules are strongly F regular .

3) All R - modules have the STPIP.

4) All injective $R$ - modules are strongly $\mathrm{F}$ - regular .

5) All injective R - modules have the STPIP .

Proof .Clear by Th. 1.15 ,Th. 1.16, Th. 2.14 and Th. 2.15 .

Recall that an $\mathrm{R}$ - module $\mathrm{M}$ is said to has the PIP if the intersection of any two pure sub modules of $M$ is again pure, [3] . 
Theorem 2.17 [3] Let $\mathrm{R}$ be a ring . The following statements are equivalent :

1) $R$ is a regular rings .

2) All R - modules have the PIP .

3) All injective $R$ - modules have the PIP.

Now, we show by an example that an $\mathrm{R}$ - module that has the PIP, may not have the STPIP.

\section{Example 2.18.}

Let $\mathrm{R}$ be a regular ring which is not semisimple . By Th. 2.16, there exist a module $M$ such that $M$ does not have the STPIP. By Th.2.17, M has the PIP.

\section{References :}

1. Naoum, G. 1995. Regular multiplication modules, Period. Math. Hungarica.31:102-155.

2. Tütüncü, D. K. , Ertas, N. O. ,Tribalc, R.and Smith, P.F. 2011 .On Fully idempotent modules, comm. Algebra, 39:2707-2722.

3. Naoum ,A. G. , AL - HashimiB. A. and AL - Bahrani, B. H.. Modules with the pure intersection property I , submitted .

4. Kasch ,F. 1982 . Modules and Rings ,Acad . press , London .

5. Goodearl ,K.R. 1976 . Ring Theory , Nonsingular Rings and Modules Marcel Dekker, New York .

6. Naoum,A.G. 1996. 1/2 Cancellation modules, Kyung pook Math.J. ,36:97-106.

7. Garcia J.L. 1989. Properties of direct summands of modules, comm. Algebra.

8. Clark ,J. ,Lamp , C., Vanaja, N., Wisbauer, R.. Lifting modules, Supplements and projectivityin Modules Theory .Frontiers in Mathematics, Basel - Boston .

9. Mijbass ,A.S. 1997.Quasi Dedekind modules, Ph.D. Thesis, College of Science University of Baghdad.

10. Rotman ,J.J. 1979. An introduction to homological algebra, Academic Press, New York.

11. Abbas ,M.S.. 1991.On fully modules, Ph.D. Thesis, College of Science University of Baghdad .

\section{المقاسات قوية النقاء من النمط F وخاصية التقاطع قوي النقاء}

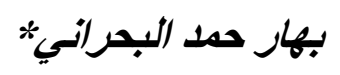

* جامعة بغداد ، كلية العلوم ، قسم الرياضيات

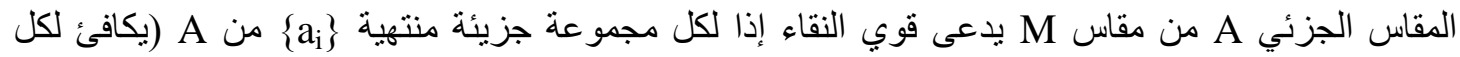

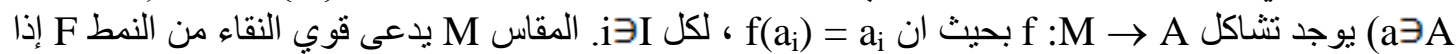

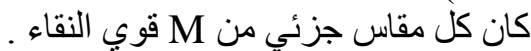

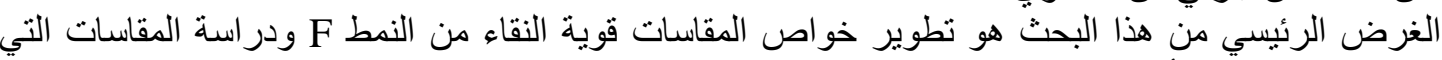
تحقق خاصية تقاطع أب مقاس جزئيين قويين النقاء يكون قوي النقاء لنقاء. 